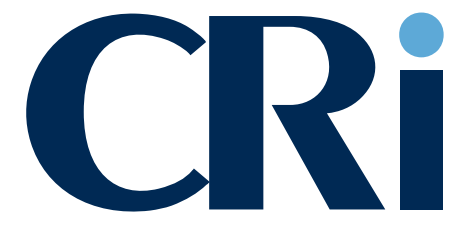

Issue 1

15 February 2008

P. 1-32
A Journal of Information Law and Technology

\title{
Articles
}

Christoph Antons - National Bans for YouTube: The Debate in Thailand in Comparison with India, Turkey and Germany

Marco Gercke - National, Regional and International Legal Approaches in the Fight Against Cybercrime

John P. Beardwood/Andrew C. Alleyne - Open Source Hybrids and the Final GPLv3

\section{Case Law}

USA: Jurisdiction And Damages Against UK Based Spam Watchdog e360 Insight v. The Spamhaus Project

Canada: Declaration of Non-Infringement Research in Motion Ltd. v. Atari Inc. et al.

\section{Updates}

Neil Wilkof/Karen L. Elburg/ Shir Uzrad - Israel: Proposed Internet Censorship Legislation

Probir Roy Chowdhury - India: Interim Order Against Copyright Violation on YouTube.com

Sami Sunila - Finland: Copyright Council's Opinion on TPM Effectiveness

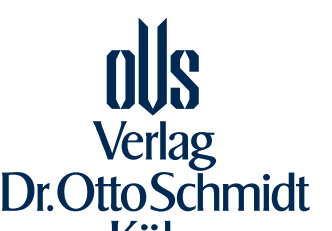

Köln 


\section{Wir beamen Sie auf den

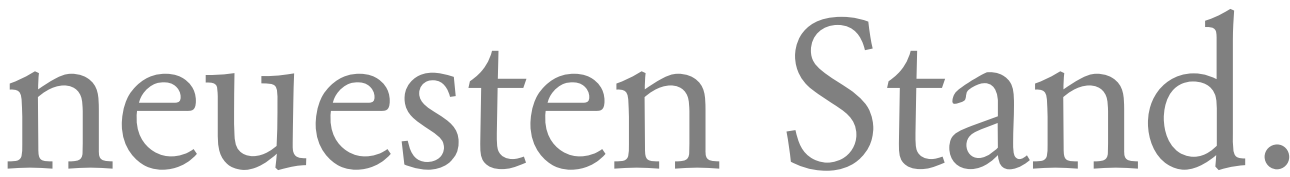

Die zweite, komplett überarbeitete Auflage dieses begehrten Handbuchs zum Telekommunikationsrecht bringt Sie rundum auf den allerneuesten Stand. Die weitreichenden Änderungen durch das TKG-Änderungsgesetz 2007 sind bereits in vollem Umfang eingearbeitet.

Das Buch schafft endlich wieder Klarheit im Regelungs- und Regulierungsdickicht der unübersichtlichen Materie, die sich seit der Vorauflage grundlegend verändert hat. Zum einen durch die vielen Novellierungen des TKG und seiner Nebengesetze. Zum anderen durch die vielen Folgeprobleme, mit denen die Gesetzesanwendung Sie heute konfrontiert.

Ging es früher vor allem um Rechtsfragen im Zusammenhang mit der Installation des Leitungsnetzes, stehen jetzt die Probleme der

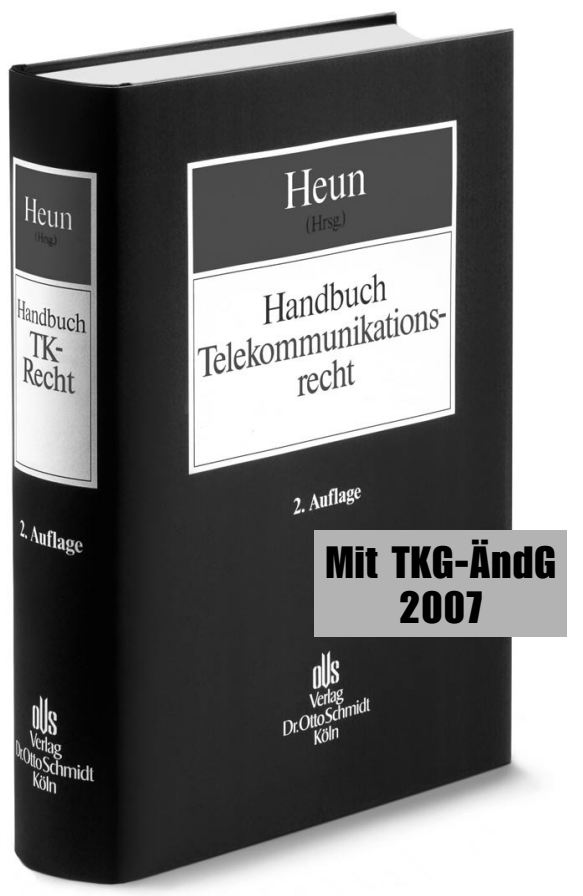

Heun (Hrsg.) Handbuch Telekommunikationsrecht Herausgegeben von RA Sven-Erik Heun. Bearbeitet von sechs praxiserfahrenen Autoren. 2., vollständig überarbeitete Auflage 2007, 1.664 Seiten Lexikonformat, gbd. $169,-€$. ISBN 978-3-504-56028-7
Markt- und Entgeltregulierung und des Kundenschutzes im Vordergrund.

In zwölf übersichtlich gegliederten Teilen beantworten Ihnen die praxiserfahrenen Autoren systematisch alle Fragen, mit denen Sie als Berater im Telekommunikationsrecht rechnen müssen. Detailliert, aber praxisrelevant gewichtet. Alle Lösungsvorschläge sind mit konkreten Handlungsempfehlungen verbunden und orientieren sich allein an den Bedürfnissen der Praxis. Die Autoren schreiben völlig unabhängig von Anbieterinteressen, allein aus Sicht des anwaltlichen Beraters.

Das Handbuch Telekommunikationsrecht beamt Sie im Handumdrehen auf den neuesten Stand. Sie müssen es nur bestellen. Leseprobe? www.otto-schmidt.de

Bestellschein ausfüllen und faxen (02 21) 937 38-9 43 조요

X Ja, ich bestelle mit 14-tägigem Rückgaberecht Heun (Hrsg.) Handbuch Telekommunikationsrecht 2. Auflage, gbd. 169,- € plus Versandkosten. ISBN 978-3-504-56028-7 
New at www.cr-international.com

UK - IPO proposal of 8 January 2008

to Copyright Exception

\section{Computer Law Review Internationa1}

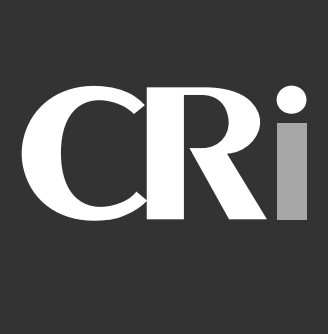

A Journal of Information Law and Technology

9th Year $\cdot$ Issue 1/2008 · Pages 1-32

\section{Articles}

\section{Editorial Board}

Prof. Dr. Thomas Dreier, M.C.J

University of Karlsruhe

Dr. Jens-L. Gaster, principal administrator, Brussels

RA Thomas Heymann, Frankfurt/M.

Prof. Dr. Michael Lehmann, Dipl.-Kfm

Max-Planck-Institute and University of Munich

Prof. Raymond T. Nimmer, University of Houston

Attorney at Law Holly K. Towle, J.D., Seattle

Attorney at Law Thomas Vinje, Brussels
Christoph Antons - National Bans for YouTube: The Debate in Thailand in Comparison with India, Turkey and Germany

Marco Gercke - National, Regional and International Legal Approaches in the Fight Against Cybercrime

John P. Beardwood/Andrew C. Alleyne - Open

Source Hybrids and the Final GPLv3

Decision of 30 August 2007

Canada: Declaration of Non-Infringement -

Research in Motion Ltd. v. Atari Inc. et al. - Ontario Superior Court of Justice - Decision of 16 August 2007

USA: Enforceability of Foreign Judgments in the US - Sarl Louis Feraud International et al. v. Viewfinder, Inc. - Court of Appeals for the $2^{\text {nd }}$ Circuit Decision of 5 June 2007

\section{Updates}

Neil Wilkof/Karen L Elburg/ Shir Uzrad - Israel: Proposed Internet Censorship Legislation

Probir Roy Chowdhury - India: Interim Order Against Copyright Violation on YouTube.com

Sami Sunila - Finland: Copyright Council's Opinion on TPM Effectiveness

- About the Authors

- Masthead

- CRi-Reader Services 7 14

\section{Case LaW}

USA: Jurisdiction And Damages Against UK Based Spam Watchdog - e360 Insight v. The Spamhaus Project - Court of Appeals for the $7^{\text {th }}$ Circuit -

\author{
Awocato Pietro Tamburrini (ltaly) \\ Attorney at Law Thomas Vinje (USA, EU) \\ Prof. Coenraad J. Visser (South Africa) \\ J.T. Westermeier (USA) \\ Neil J. Wilkof (Israel) \\ Jamie Wodetzki (Australia) \\ Attorney at Law Sakari Aalto (Finland)
}



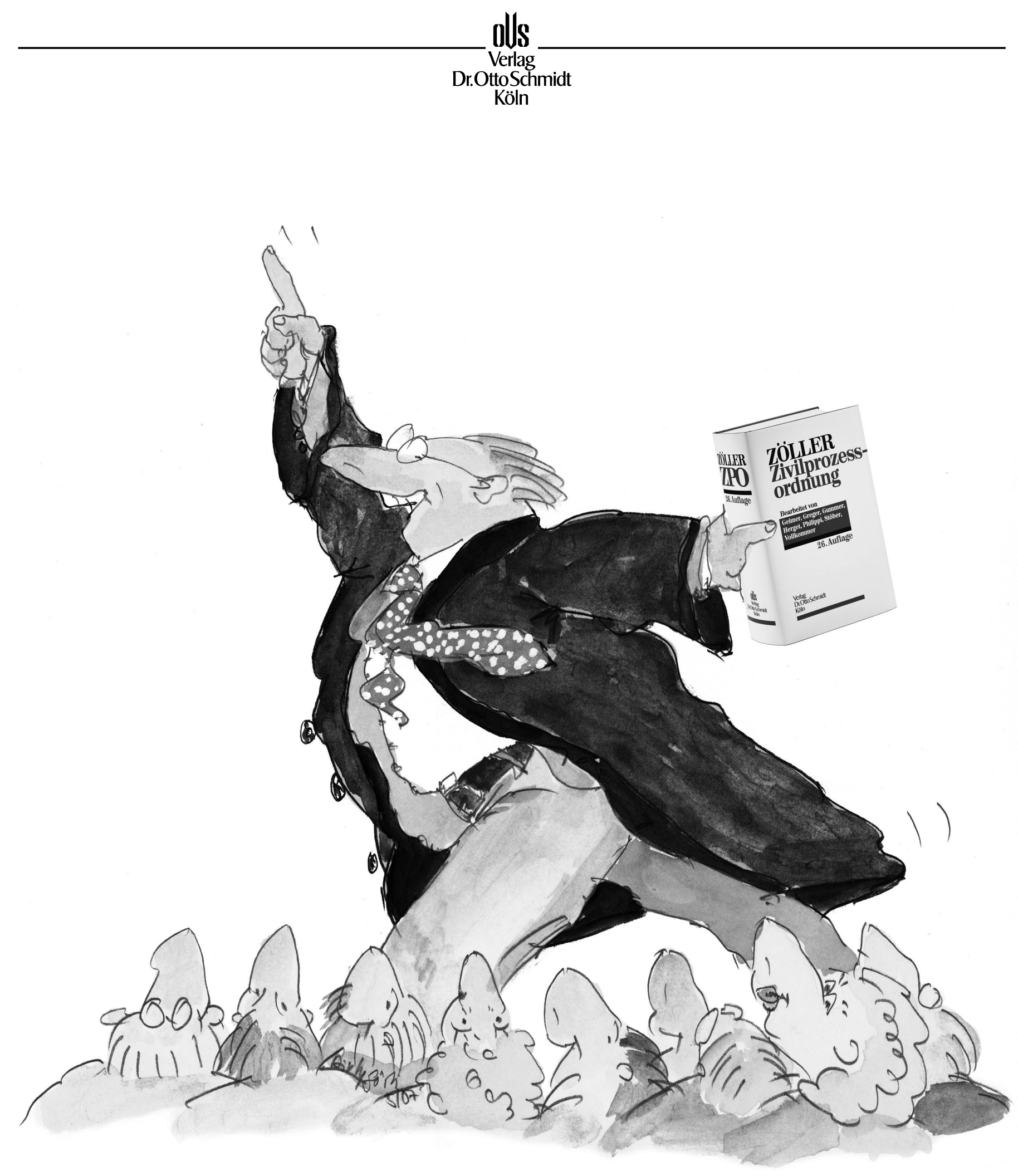

\section{Kein Prozessrecht ohne Zöller.}

Auch nach 26 Auflagen zeigt der Zöller, wo es im Zivilprozessrecht langgeht. Unveränderter Autorenkreis. Unverändert hohe Qualität. Zöller, Zivilprozessordnung, 26., neu bearbeitete Auflage 2007, gbd. 154,80 €. ISBN 978-3-504-47015-9. Bestellen Sie bei Ihrer Buchhandlung oder bei www.der-zoeller.de 